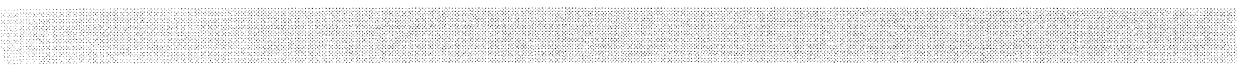 \\ Praxisberichte
}

\section{Supervision in der Gesundheitsförderung}

\author{
Heidi Möller, Berlin
}

Mit Supervision wird üblicherweise der psychosoziale Kontext assoziiert; Supervision im prätherapeutischen Raum, dem die Gesundheitsförderung zuzuordnen ist, ist heute eher noch eine Seltenheit. In der Gesundheitsförderung haben wir keinen expliziten Behandlungsauftrag durch die uns anvertraute Klientel. Dennoch sind wir Diener und Pfleger (Übersetzung des griechischen Begriffs ,Therapeut“) der Gesundheit, z.B. der Kursteilnehmerinnen von Präventionsangeboten. Damit ist ein Spannungsfeld eröffnet, in dem sich Kursleiterinnen oft genug als Grenzgängerinnen zwischen heilenden und funktional übenden Interventionen erleben. Ich möchte hier die Möglichkeiten und Notwendigkeiten von Supervision in der Gesundheitsförderung diskutieren.

\section{Supervision in der Gesundheitsförderung}

In der Supervision unterscheiden wir verschiedene Settings, in denen der Beratungsprozess stattfindet. Einzelsupervision als Coaching oder Rollenberatung stellt die Methode der Wahl für Leiterinnen von Instituten der Gesundheitsförderung dar (vgl. Schreyögg 1995). Der Fokus dieses Aufsatzes liegt jedoch bei den Möglichkeiten von Supervision der Arbeit der in der Gesundheitsförderung tätigen Kursleiterinnen. Hier stellt Supervision eine Zwitterstellung zwischen Gruppen- und Teamsupervision dar. Es handelt sich weder um eine Gruppensupervision, die dadurch definiert ist, dass die Supervisandinnen (1) keine berufliche Beziehung zueinander haben, (2) sich zunächst nicht kennen, (3) aus unterschiedlichen Praxisfeldern stammen und (4) sich freiwillig in einer Gruppe zusammenfinden, um sich in Bezug auf ihre Rolle und ihr professionelles Handeln einem Reflexionsund Qualifizierungsprozess zu unterziehen; noch handelt es sich um klassische Teamsupervision, die zur Voraussetzung hat, ,,dass alle Mitglieder der Teamsupervision (...) Mitarbeiter sind, die in einer Institution arbeiten und ein gemeinsames Aufgabengebiet gegen Entlohnung kooperativ bewältigen sollen" (Conrad, Pühl 1983, 85).

Kursleiterinnen arbeiten zwar an der gemeinsamen Sache der Gesundheitsförderung, z.B. in der Stressbewältigung, jedoch sind sie in der Regel Einzelkämpferinnen, die in der Arbeit mit ihren Gruppen allein stehen. Sie haben anders als Teams keine Organisationsberatung - Supervision - Clinical Management, Heft 2/2000, S. 145-155 
gemeinsame Klientel, jedoch ähnlich gelagerte Aufgaben. In ihrem beruflichen Handeln sind sie allein. Der Supervision kommt hier eine identitätsstiftende Funktion zu. Sie kann leisten, dass sich die Kursleiterinnen einer gemeinsamen Sache verpflichtet und sich der sie entlohnenden Institution im Sinne einer „corporate identity“ zugehörig fühlen. Dies erleben die Supervisandinnen innerhalb der Gesundheitsförderung als unterstützend. Sie sind oft darauf angewiesen, an unterschiedlichen Stellen für ihren Lebensunterhalt zu sorgen, und ihnen fehlt der professionelle Halt einer Institution, die Festanstellungen der Mitarbeiterinnen ermöglicht.

\subsection{Probleme der Supervision in der Gesundheitsförderung}

Supervision hat immer pädagogische Effekte (vgl. Pühl 1992). In der Ausbildungsund Fortbildungssupervision findet eine Qualifizierung statt. Dieser Aspekt erschein mir insbesondere deshalb als bedeutsam, weil das Arbeitsfeld der Gesundheitsförderung jungen Kolleginnen die Möglichkeiten eines beruflichen Einstiegs bietet. Gesundheitsförderung (zumeist als Kurse angeboten) findet oft mit Hilfe strukturierter Kursmanuale statt, die gerade Neulingen ein hohes Maß an Sicherheit stiften. Die einzelnen zum Teil recht schnell entwickelten Programme stehen unter Erfolgsdruck, und ein hoher, möglichst mit großen Teilnehmerinnenzahlen und Erfolgsmessungen unterfütterter Output wird immer wichtiger.

Es ist zu beobachten, dass die Teilnehmerinnen an Kursen der Gesundheitsförderung schwerere Pathologien aufweisen. Waren früher leichte funktionelle Störungen der Anlass, Gesundheitsförderung nachzusuchen, so sind heute Patienten mit schweren Psychosomatosen, die den Weg in die Psychotherapie scheuen, die übliche Klientengruppe. Es finden sich z.B. Menschen in den Programmen gegen Rückenschmerzen, die wohlwollend dem Bereich tertiärer Prävention zuzuordnen wären. Gesundheitsförderung hat hier einen wichtigen Auftrag, denn die Teilnehmerinnen sind für ein eigentlich psychotherapeutisches Angebot oft nicht zugänglich. Auf der anderen Seite wissen erfahrene Psychotherapeutinnen, dass die Arbeit mit manifesten Psychosomatosen eine der schwierigsten Aufgaben ist. Z.B. haben gerade Schmerzpatientinnen zum einen mit die schlechteste Prognose und zum zweiten die niedrigste Motivationslage zur Verhaltensänderung. Kursleiterinnen brauchen an dieser Stelle eine Nachqualifizierung im Sinne von Ätiologie und Behandlungsansätzen, um ihrer Aufgabe gerecht werden zu können. Eine angemessene individuelle Zielfindung sowohl für die Klientel als auch für die Mitarbeiterinnen ist sinnvoll, um Misserfolgen und Frustrationserlebnissen auf beiden Seiten vorzubeugen. Supervision in der Gesundheitsförderung nimmt m.E. eine Zwitterstellung zwischen Aus- und Fortbildungssupervision ein, denn wenige Kursleiterinnen sind speziell für ihre Arbeit im prätherapeutischen Raum ausgebildet.

Ich möchte auf eine der wesentlichen Wurzeln heutiger Supervisionspraxis verweisen. Michael Balint entwickelte seine Methodik als Didaktik für Hausärzte in Seminarform. Es ging ihm um die emotionale Verarbeitung von Praxiserfahrung mitsamt einer Vermittlung von Theorie zum besseren Verständnis der Problematiken des Arbeitsalltags. Ohne den Fortbildungsaspekt der Supervision verkäme diese zu einem gemeinsamen „Wiederkäuen“. Ein Lerninput kann m.E. zu Recht erwartet werden. Der Supervisionsprozess ist eine Lernerfahrung. Damit sind innere und äußere Bewertungen verbunden. Die Qualifikation der Kursleiterinnen wird quasi öffentlich, die Gruppenmitglieder und die Supervisorin machen sich ein Bild, die Arbeit wird eingeschätzt. Viele Teilnehmerinnen von Supervisionsgruppen fühlen sich, als ob sie auf dem Prüfstand 
stünden. Ihre kognitiven, intellektuellen und Erlebensdimensionen werden sichtbar. Die Angst vor Schamgefühlen (vgl. Möller 1998) und/oder vor Kritik in Supervisionsgruppen ist groß.

Der Haltung der Supervisorin kommt hier eine besondere Bedeutung zu. Sie befindet sich in einer Position der Autorität und der besonderen Kennerschaft. Sie nimmt die Beziehungsdynamik der Kursleiterinnen zur Klientengruppe und/oder zu einzelnen Teilnehmerinnen wahr, sieht blinde Flecken oder notorische Wahrnehmungsverzerrungen der Kursleiterinnen. Diese zu benennen und im günstigen Fall aufzulösen, erhöht die Effizienz der Arbeit. Es erscheint mir wichtig, dass die Supervisorin sich potentiell überflüssig macht. Die Kursteilnehmerinnen sollen eine eigene Fähigkeit zur Reflexion erwerben, auf Dauer ihr eigener Supervisor werden können, d.h. supervisorisches Tun verinnerlichen, über Widersprüche nachdenken, Konzepte überdenken, kritische Distanz zu vorgegebenen Manualen zu bewahren u.v.m. Die Position der Supervisorin sollte nie die der Besserwissenden, sondern stets die der Anderswissenden sein (vgl. Haesler 1996). Nur die Freiheit des Nachdenkens, wo kontroverses Reflektieren und Handeln möglich ist und Kreativität seinen Raum hat, schafft ein fehlerfreundliches Klima und wirkt der Entmündigung der Kursleiterinnen entgegen. Die Supervisorin bietet Relativierungen und Vermutungen an, versucht die Bedeutungen bestimmter Phänomene zu entdecken und zu verstehen. Sie weiß dann (hoffentlich) nicht besser, sondern mehr.

So ist es m.E. wichtig, vordergründig sich als Fehler präsentierende Verhaltensweisen von Kursleiterinnen als Reaktionen auf die bewussten, vorbewussten oder unbewussten Aktivitäten der Gruppen der Kursteilnehmerinnen zu verstehen. Dynamische Aspekte der Gruppenprozesse der Teilnehmerinnen zeigen sich im Erleben und Verhalten der Leiterinnen. Solchen Phänomenen sinnverstehend zu begegnen und mit den Supervisandinnen eine Übereinstimmung ihres Erlebens mit den Gruppenlatenzen, d.h. den unbewussten Phantasien der Gruppe und/oder gruppenspezifischen Abwehrformationen der Teilnehmerinnen herzustellen, kann ein tragfähiges Lernbündnis schaffen. Es ist oft eine Erleichterung, wenn Kursleiterinnen ihre Gefühle in Gruppen zu verstehen lernen, wenn sie den Druck, unter dem sie häufig stehen, mit der Gruppendynamik der Teilnehmerinnen in Zusammenhang bringen können. Die Gesundheitsförderung würde eine Menge Möglichkeiten der Verbesserung gesundheitsbildnerischen Tuns verschenken, wenn sie auf die Unterstützung der Arbeit durch Supervision verzichtete.

Mir scheint es sinnvoll und notwendig, Empfehlungen über Interventionen zu geben, ohne die Kursleiterinnen übermäßig von ihrer Aufgabe zu entlasten und damit zu entmündigen. Es gilt, fachliche Empfehlungen zu geben, ohne den eigenen Entscheidungsdruck von den Supervisandinnen fernzuhalten. Voraussetzung dafür ist die notwendige Spannungstoleranz der Supervisorin. Sie muss gelernt haben, eigene Angst in Gruppen und Institutionen wahrzunehmen und zu handhaben, damit sie sich nicht zu einem vorschnellen Eingreifen, zu Strukturierungshilfen und fachlichen Kommentaren hinreißen lässt. Es besteht die Gefahr der Entmündigung der Supervisandinnen, ihnen zuviel Verantwortung abzunehmen und damit Träger von Idealisierungen zu werden.

Sicher verfügt die Supervisorin über Macht und Einflussnahme; sie sollte jedoch auf keinen Fall zum kritischen Über-Ich der Institution werden. Davor ist die Supervisorin umso besser gefeit, je mehr sie über die eigenen Abwehrmanöver weiß. Daneben ist die Supervisorin ein Modell, von dem man lernen, an dem man sich orientieren und von dem man sich abgegrenzen kann. Die Haltung der Supervisorin kann Vorbildfunktion für einzelne Supervisandinnen sein. Durch Identifizierung und partielle identifika-

Organisationsberatung - Supervision - Clinical Management 
torische Übernahme von Haltungen werden Interventionstechniken und Gruppenleitungsfunktionen quasi ,eingeschliffen“.

Es besteht die Gefahr einer übermäßigen Idealisierung der Supervisorin. Ihre Werte, Anschauungen und Haltungen sollten sicherlich nicht unkritisch übernommen werden. Sich als Projektionsfigur zur Verfügung zu stellen, kann in gewissem Maße als durchaus förderlich beschrieben werden. Lernen funktioniert $\mathrm{zu}$ wesentlichen Teilen durch die „Einverleibung“ der Lehrenden. Neue Orientierungen werden möglich, das Risiko, etwas bisher Tragendes in Frage zu stellen, kann in anderer Weise eingegangen werden. Supervision findet nach Möglichkeit „,durch einen erfahrenen Fachmann statt, der über besondere Kompetenzen verfügt" (Pühl 1992, 2).

Das heißt, Supervision geschieht immer in einer Interaktion mit Kompetenzgefälle, und das scheint mir mehr als nur ein legitimes Recht der Nachfragenden nach Supervision. Symmetrische Beziehungsgestaltung (vgl. Schreyögg 1991) mag dabei eine Zielmarke sein, ist in der konkreten Arbeit jedoch als illusionär zu bezeichnen. Der Erfahrungs- und Wissensvorsprung lässt die Supervisionsbeziehung immer leicht asymmetrisch werden. Sicher versteht eine gute Supervisorin sich immer auch als Lernende; aber es ist ihr größeres Erfahrungswissen, das sie ihr Geld wert sein lässt. Supervisorinnen in der Gesundheitsförderung sollten Lehrerinnen sein, ohne zu dozieren, und eine positive Autorität verkörpern, ohne zu entmündigen.

\subsection{Die Supervisorin als Leitungsfigur in der Gesundheitsförderung}

Im Folgenden werden Vignetten gezeigt, in denen die Supervisorin auch eine Leitungsfunktion übernimmt. Die Zusammenstellung ist sicherlich ergänzungsbedürftig und erhebt keinen Anspruch auf Vollständigkeit.

(1) Gerade in Arbeitsfeldern wie der Gesundheitsförderung, in denen das Personal häufig wechselt, die viel mit Honorarkräften auskommen müssen, kommt der Supervisorin eine besondere Rolle zu. Sie schafft die Kontinuität, die die Institution nicht bereitstellen kann. Oft muss sie mit ihrer Person und ihrem Angebot wie ein Fels in der Brandung gegen Mitarbeiterfluktuation, Abwesenheit von Kursleiterinnen, Auftauchen und Verschwinden von Praktikantinnen stehen.

(2) Fachaufsicht und Kontrolle stellen die Ursprünge des Supervisionsbegriffs in den USA dar. Diese Tatsache ist auf der einen Seite mitverantwortlich für den Schrecken, den der Begriff Supervision bei Nachfragern dieser Dienstleistung auslöst. Auf der anderen Seite macht es Sinn, sich auf die Wurzeln zu berufen, denn viele Leiter von Instituten der Gesundheitsförderung sind zwar in der Lage, die Dienstaufsicht zu gewährleisten, haben aus zeitlichen Gründen oft keine Kapazitäten für die fachliche Aufsicht ihrer Mitarbeiter. Ihre beruflichen Kompetenzen liegen im Zuge der Verschiebung der Arbeitsprofile hin zur Wahrnehmung von Managementaufgaben oft in anderen Bereichen als die ihrer Mitarbeiter. So ist es ihr legitimes Interesse, Qualitätskontrolle der fachlichen Arbeit, z.B. der Kursleiterinnen in Gesundheitsförderungsinstituten, in die Hände einer Supervisorin zu legen.

(3) Die Verknappung der Gelder im Bereich der Gesundheitsförderung macht die Verlagerung bestimmter Aufgaben nach außen (in diesem Fall zur Supervisorin hin) notwendig, da der gekürzte Stellenpegel mit einer Streichung von Aufgaben einhergehen muss, um der Überforderung von Leitungskräften entgegenzuwirken. So wird Supervision z.B. bei der halbwegs verträglichen Umsetzung von Sparbeschlüssen benötigt Dies ist eine Aufgabe, der sich die Supervision m.E. nicht entziehen sollte, wenngleich 
an dieser Stelle ethische Überlegungen greifen sollten. Supervision findet gerade heute nach der Streichung des $\S 20$ SGBV nicht in einem expandierenden psychosozialen Feld statt, sondern in einem von massiven Streichungen bedrohten Bereich, und Supervisorinnen können sich hier nicht auf eine vermeintlich neutrale Position zurückziehen, als ginge sie dies nichts an.

(4) Neben der Kontrollfunktion ist die Fürsorgepflicht der Supervisorin zu sehen. Anerkennung und Wertschätzung der Kursleiterinnen in ihrer Arbeit mit zum Teil schwierigen Kursteilnehmerinnen sehe ich als eine wichtige Burnout-Prophylaxe und als notwendig zum Motivationserhalt. Gerade im Prozess knapper werdender Ressourcen, der mit einer erheblichen emotionalen Belastung einhergeht, fehlt der Organisationskultur oft jegliche Freundlichkeit, Höflichkeit und ein tragendes Miteinander. Dort gerade sollte die Supervisorin entgegenwirken, um ein förderliches, der Idee der Gesundheitsförderung entsprechendes Klima zu erhalten und durch ihre Person eine Gegenkultur modellhaft zu repräsentieren. Der Supervision kann in solchen Institutionen eine holding function im Sinne Winnicotts zukommen. Es kann Sinn machen, sich für eine begrenzte Zeit für die Institution als Elternsubstitut zur Verfügung zu stellen. Der Wunsch von Kursleiterinnen nach Betreuung stellt auf der einen Seite ein Spiegelphänomen $^{1}$ einer schwieriger werdenden Klientel dar, die zunehmend mehr versorgt, bemuttert und wenig konfrontiert werden will. Auf der anderen Seite ist es ein reales, legitimes Bedürfnis der Kursleiterinnen nach emotionaler Unterstützung ihrer mühsamen Tätigkeit.

(5) Ebenso denkbar ist die Verkörperung von „Ersatzmanagement auf Zeit“ für die Supervisorin. Institutionen können in Krisenzeiten auf die Führung durch Supervisoren zurückgreifen und sie mit deren Hilfe bewältigen lernen. Auch strukturelle Verbesserungsvorschläge, die die Supervisorin aus der Fallarbeit oder Gruppendynamik in den Supervisionssitzungen gewonnen hat, sollten der Institution nicht verloren gehen. Regelmäßige Gespräche in diesem Sinne mit der Institutsleitung, die die Supervisorin schließlich bezahlt, halte ich für unabdingbar. Wichtig scheint mir jedoch die Transparenz dieser Gespräche den Supervisandinnen gegenüber, damit das Vertrauensverhältnis in den Supervisionssitzungen nicht beeinträchtigt wird.

\section{Zur Methodik der tiefenpsychologisch orientierten Supervision in der Gesundheitsförderung}

Im Folgenden soll supervisorisches Handeln in der Gesundheitsförderung gemäß der Methodik, der ich mich verpflichtet fühle, veranschaulicht werden.

\subsection{Die Vorbereitung}

Es ist unabdingbar, zunächst die latenten Aufträge für die Supervisorin kennenzulernen und die latente Motivationsstruktur der Mitarbeiterinnen zu erkunden. Die Supervisorin muss sich ein Bild über das komplexe Beziehungsgefüge der Gruppe der Kursleiterinnen machen, über den Zustand des „Gruppen-Ichs“, die Rivalitäten untereinander, Bündnisse und Cliquenbildungen wahrnehmen, die Beziehungen zu Vorge-

1 Reinszenierung der unbewußten Phantasien der Kursteilnehmerinnen in der Gruppe der Supervisandinnen.

Organisationsberatung - Supervision - Clinical Management 
setzten und zur Verwaltung eruieren, Institutions- und Teammythen und -phantasien aufspüren, Problemlöseverhalten der Kursleiterinnen und informelle Macht- und Hierarchiestrukturen kennenlernen und vieles andere mehr. Neben den unbewussten Arbeitsbeziehungen sind durchaus Elemente für die Supervision entscheidend, die dem rationalen Dialog zugänglich sind. Zu nennen wären z.B. unterschiedliche Vorstellungen und Erwartungen an den supervisorischen Prozess, Kursbeschreibungen, aktuelle Arbeitssituationen, die Aufgabenspezifität, die Geschichte der Institution, die berufliche Sozialisation der Kursleiterinnen etc.

Für viele Kursleiterinnen ist die Arbeit in der Gesundheitsförderung ein Einstieg in den beruflichen Alltag. Das liegt zum einen daran, dass Gesundheitsförderung gesellschaftlich eine recht neue Aufgabe ist bzw. war, zum anderen daran, dass Gesundheitsförderung einer der wenigen expandierenden psychosozialen Sektoren war, in dem junge Kolleginnen eingestellt werden konnten. Viele Kursleiterinnen befinden sich in einer Art Warteschleife, sind auf der Suche nach einer Festanstellung. Das bedeutet, dass sie jederzeit abspringen können, und dies wiederum bedeutet eine Schwierigkeit für die Gruppenkohäsion. Der Supervisor hat es u.U. ständig mit einer neuen Gruppenzusammensetzung zu tun. Es besteht die Gefahr der Entwertung der Kursleitertätigkeit, da Kursleiterinnen u.a. meinen, das eigentlich psychotherapeutische Handeln sei das Wertvollere. Dieser Wertung gilt es entgegenzuwirken.

\subsection{Der Supervisionsprozess}

In der Supervision sind alle Beziehungen und Beziehungsaspekte eingeschlossen, die sich mittelbar oder unmittelbar auf die berufliche Tätigkeit beziehen. Der fallbezogene Ansatz, etwa nach dem Muster der von Balint (1955) entwickelten Methode, und die konfliktbezogenen, auf die unbewusste Gruppendynamik gerichteten Methoden sind zu unterscheiden als zwei Perspektiven und damit als Teile ,der psychoanalytischen auf die unbewussten Beziehungen gerichteten Wahrnehmungseinstellung und der daraus resultierenden Disposition zu einer deutenden Aktivität“" (Becker 1995, 9) zu sehen. Die unbewusste Beziehung zwischen Kursteilnehmerinnen und Kursleiterinnen wird in die Supervisionssitzung transponiert und kann dort spürbar und erlebbar werden. Dafür bildet die szenische Funktion des Ich des Supervisanden (Argelander 1970) die Voraussetzung. Seine unbewusste Beziehung zur Teilnehmerin, seine Gegenübertragungsreaktionen inszenieren sich in spezieller Weise in der supervisorischen Situation.

Der tiefenpsychologisch orientierte Prozess der Supervision besteht nun darin, die Beziehung zwischen Kursleiterinnen und Teilnehmerinnen im Raum der Beziehung zwischen Supervisionsgruppe und Supervisorin zu analysieren und szenisch - im Sinne Lorenzers (1970) - zu verstehen (vgl. Pollak 1995, 59ff.). In der Supervision von Kursleiterinnen wird zwischen mehreren Beziehungsebenen unterschieden:

- die Beziehung der Kursleiterinnen zu den Klienten,

- die Beziehung der Kursleiterinnen untereinander,

- die Beziehungen des Kursleiterinnen-Teams zu Personen und Gruppen in der Institution.

Priorität bei der Arbeit erhält die Beziehung und deren Störung der Kursleiterinnen zu den Kursteilnehmerinnen. Die beiden anderen Ebenen werden dann wichtig, wenn sie den Arbeitsauftrag erschweren und behindern oder, wie ich ergänzen möchte, wenn die Problematik der Kursteilnehmerinnen nur mit Hilfe des institutionellen Kontextes 
als „,indirektes Spiegelphänomen“ (Kutter 1992) verstehbar ist, wenn etwa die Problemstellung in der Supervision Ausdruck teaminterner oder institutioneller Konflikte ist.

\section{Beispiel 1:}

In einem Kursdurchgang nahmen die Teilnehmerinnen eines Übergewichtsprogramms anders als in der Vorzeit überrraschenderweise nicht mehr ab. Die Institution befand sich in einer Krise, die zunächst die Kursleiterinnen verunsicherte. Sie fürchteten um ihren Arbeitsplatz. Die Angst wurde durch die in Krisen übliche Gerüchtebildung so verstärkt, dass die Kursleiterinnen ihrer Gruppe nicht mehr den schützenden Rahmen und die Sicherheit gaben, die sie zur Gewichtsreduktion nötig gehabt hätten. Die Existenzängste der Institution und ihrer Mitglieder reinszenierte sich in der Teilnehmerinnen-Gruppe, die mit unbewusstem Widerstand, der Verweigerung, Gewicht zu verlieren, antwortete.

Die Kunst der Supervisorin besteht nun darin, zwischen Reaktionen der Gruppe auf den Fallbericht, gruppeninternen Konflikten und Konflikten der Gruppe mit der Institution zu unterscheiden bzw. das Ineinanderwirken der Faktoren zu analysieren. Ihre Aufgabe ist es somit, u.a. direkte von indirekten Spiegelphänomenen zu unterscheiden. Dazu kann die Supervisorin nicht anders, ,,als in der Supervisionsgruppe auch den Gruppenprozess wahrzunehmen“ (Kutter 1992). Ziel der Supervision ist die Übertragungsdynamik zwischen Teilnehmerin und Kursleiterin aufzudecken und den latenten Sinngehalt der unbewussten Beziehungsnahme zu verstehen. Daraus kann eine differenziertere Wahrnehmung der Kursteilnehmerinnen und eine adäquatere Haltung in der Behandlung erwachsen. Die Kursteilnehmerin ist während der Supervision nicht anwesend, dennoch entfaltet sich ihre Psychodynamik in der Sitzung als Spiegelphänomen (Kutter 1992).

Gegenstand der tiefenpsychologisch orientierten Supervision ist die unbewusste Übertragungs-Gegenübertragungs-Beziehung zwischen Kursleiterin und Teilnehmerin. Dabei kann die Gegenübertragung in der Gruppe von Kursleiterinnen zum einen als eine von der Kursteilnehmerin induzierte, für die Teilnehmerin charakteristische Reaktion gesehen werden, was dann Aufschluss über die Übertragung und unbewusste Erlebniswelt des Teilnehmers geben kann; zum anderen kann sie als eine für die Supervisionsgruppe charakteristische Reaktion auf eine Kursteilnehmerin aufgefasst werden, wodurch die Supervisorin eher Aufschluss über das Unbewusste der Gruppe erhält. Wenn hier von Gegenübertragung die Rede ist, dann ist das insofern eine Vereinfachung, als die einzelnen Gruppenmitglieder natürlich nicht als psychodynamische Einheit gesehen werden können. Die jeweilige Kursteilnehmerin entwickelt gegenüber einzelnen Kursleiterinnen unterschiedliche Übertragungsfacetten, wie auch die einzelnen Kursleiterinnen unterschiedliche Gegenübertragungsaspekte entwickeln.

\section{Beispiel 2:}

Die oft sehr jungen, attraktiven und vor allem schlanken und beweglichen Kursleiterinnen von Übergewichtsprogrammen evozieren eine ganz andere Neid- und Rivalitätsdynamik bei den eher älteren und unter ihrem Übergewicht leidenden weiblichen Kursteilnehmerinnen als eine ältere eventuell selbst einmal übergewichtig gewesene Kursleiterin. Es erleichtert die Arbeit der Kursleiterinnen zu wissen, welche Übertragungen sie durch ihr spezielles Sosein auf sich ziehen. Es macht sie handlungsfähiger, und sie zeigen sich gewappneter in kritischen Phasen des Gruppenprozesses.

In der unbewussten Übertragungs-Gegenübertragungs-Beziehung zwischen Supervisionsgruppe und Supervisorin spiegelt sich die Dynamik von Kursteilnehmerinnen ebenfalls wieder. Dabei lassen sich drei Ebenen unterscheiden: 
(1) Die privaten Übertragungen der Gruppenmitglieder auf die Supervisorin und untereinander sowie die privaten Gegenübertragungen der Supervisorin auf die Teilnehmerinnen, die nicht Gegenstand der Supervision sind.

(2) Übertragungen der Gruppe auf die Supervisorin als Gruppenleiterin mit der entsprechenden Gegenübertragung der Supervisorin auf die Supervisionsgruppe. Hier kommt dem Gruppenprozess in der Supervision zentrale Bedeutung zu, die Dynamik des habituellen Unbewussten der Gruppe stellt den entscheidenden Bezugspunkt dar. Alle Fragen, die die Institution und deren Mitarbeiterinnen nicht gelöst haben, stellen sich früher oder später im supervisorischen Prozess.

Beispiel 3:

Die Supervisorin begrüßt einen neuen Kursleiter in der Supervision. Dieser attackiert sie sofort, greift ihre Kompetenz an und hält ellenlange Vorträge über die gesellschaftliche Relevanz von Gesundheitspsychologie. Keine anderere Kursteilnehmerin sagt etwas, die Atmosphäre im Raum ist hochexplosiv gespannt. Nach mehreren Kooperationsangeboten seitens der Supervisorin erfolgt kein Entgegenkommen des neuen Gruppenmitglieds. Ein Konsens über gemeinsames Arbeiten auf der Fallebene, jenseits theoretischer Ausführungen, ist nicht herzustellen. Die Supervisorin bittet den Kursleiter, die Supervision zu verlassen, da sie sich außer Stande sieht, mit ihm zu kooperieren. Die anderen Mitglieder der Supervisionsgruppe sind sichtbar erleichtert. Sie sehen sich befreit von dem Druck, dem sie sich seitens der Institutionsleitung ausgesetzt sahen, jeden ihrer Kursteilnehmer in der Gruppe halten zu müssen. Sie können sich zugestehen, mit einzelnen Teilnehmern nicht „fertig zu werden“, so wie es auch der Supervisorin nicht gelang. Die Kursleiterbetreuerin bedankt sich Tage später überschwenglich, sie habe seit Monaten vorgehabt, den jungen Mann zu kündigen, es jedoch nicht über das Herz gebracht.

(3) Die Dimension der Gegenübertragung der Kursleiterinnen auf die Teilnehmerin, die in der Supervision in spezieller Weise erscheint und auf die die Supervisorin ihrerseits mit einer Gegenübertragung zweiter Ordnung reagiert.

\section{Beispiel 4:}

Die teilnehmenden Frauen in den Kursen der Gesundheitsförderung sind oft in dem Alter der Mütter der Kursleiterinnen. Problematische, nicht durchgearbeitete Beziehungen zu den eigenen Müttern kann die Wahrnehmung der teilnehmenden Frauen so überschatten, dass die Arbeit unmöglich wird. Die Supervisorin reagierte bei der Vorstellung einer Gruppe von vorwiegend älteren übergewichtigen Frauen mit einer eigenartigen Müdigkeit. Sie kennt diese Form plötzlich sie überfallender Müdigkeit als für sie typische Gegenübertragungsreaktion auf Themen im Zusammenhang mit Loslösungsaggressionen gegenüber der Mutter. Sicherlich ist die Müdigkeit für sich allein genommen kein ausreichender „Beweis“ für das Auftauchen einer Mutter-Tochter-Problematik. Sie kann nur ein Hinweisreiz sein, der durch die Gruppenresonanz und vor allem die der vorstellenden Kursleiterin verifiziert werden muss. Das Beispiel mag jedoch verdeutlichen, wie wichtig es für Supervisorinnen ist, die für sie typischen Gegenübertragungsreaktionen zu kennen.

\subsection{Die Technik der tiefenpsychologisch orientierten Gruppensupervision}

Die Supervisorin tastet die verschiedenen Phänomene, die in der Sitzung zutage treten, für die Deutungsformulierung danach ab, inwieweit sie als Gegenübertragung der Kursleiterinnen auf die Teilnehmerinnen verstanden werden können. Oftmals reproduziert die Gruppe der Supervisandinnen die Arbeitssituation in der Form, in dem sie sich mit den Kursteilnehmerinnen identifiziert und die Supervisorin so behandelt, wie sie sich vom Patienten behandelt fühlen. Oder: die Supervisorin wird immer mehr in die 
Rolle der Kursleiterinnen gedrängt. Für Teamsupervisionen formulierte es Argelander (1980) folgendermaßen: „,Der Supervisor wiederum ist mit dem Team identifiziert. Indem er seine eigenen Reaktionen auf das Team als Ausdruck dieser transponierten Identifikation erkennt, kann er sie deuten: Er bezieht seine Reaktionen gegenüber dem Team - als Gegenübertragung zweiter Ordnung - zurück auf die Reaktion des Teams gegenüber dem Patienten“ (zit.n. Pollak 1995, 70). In einem zweiten Schritt kann diese Gegenübertragungsreaktion auf die Lebensgeschichte der Kursteilnehmerinnen oder auf die Geschichte der Institution bezogen werden.

\section{Beispiel 5:}

Die Gruppenstimmung ist resignativ, unterschwellig rebellisch, es will nicht recht eine tragfähige Arbeitsatmosphäre entstehen. Die Supervisorin fühlt sich in die Rolle gedrängt, anzutreiben, zur Arbeit aufzufordern, viel Zuspruch und Unterstützung zu geben. Sie sieht ihre supervisorische Kompetenz in Frage gestellt und fragt sich plötzlich nach der Sinnhaftigkeit ihres Tuns Auf Nachfragen stellt sich heraus, dass die Kursleiterinnen nach einem Leitungswechsel im Präventionsinstitut sich in keiner Weise in ihrer Leistung wertgeschätzt fühlen. Es gibt nun unterschiedliche Vergütungsstufen, deren Kriterien unklar blieben, und die als kontrollierend erlebten Interventionen der Leitung nahmen vielen die Lust am Tun. Die neue Leitung stand dermaßen unter Erfolgszwang, dass sie ihn ungefiltert an die Kursleiterinnen weitergab. Die Arbeitsfreude drohte zu sterben.

Es finden „interpersonale Angleichungs-Prozesse“ statt. Becker $(1995,91)$ beschreibt es für die psychoanalytische Teamsupervision folgendermaßen: „Der Supervisor soll unbewusst zur Identifikation gedrängt werden, das Team will, dass der Supervisor den Fall gleichsam mit den Augen des Teams sieht. Diese unbewusste Absicht steht dem bewussten Bedürfnis nach Aufklärung entgegen. (...) Die wesentliche Differenz zwischen Team und Supervisor besteht nun darin, dass sich letzterer qua psychoanalytischer Kompetenz diesem Druck reflektierend zu entziehen vermag. Dadurch begibt er sich aus dem Mittelpunkt des Geschehens an die Peripherie, an einen dezentralen Standort, von dem aus er seine Interventionen formuliert." Die Supervisorin arbeitet an dieser Stelle am ehesten gemäß der klassischen psychoanalytischen Aufgabe, der Bewusstmachung des Unbewussten. Neue Perspektiven, die im supervisorischen Prozess gewonnen wurden, müssen sich nun erst einmal im Praxisfeld bewähren oder verworfen werden. Die gemeinsame Arbeit ist im günstigsten Fall ein Wechselprozess zwischen Team und Supervisor.

\section{Beispiel 6:}

Die Kursleiterinnen von Hypertonikerprogrammen fühlten sich oft ausgelaugt nach den Sitzungen. Sie zweifelten an ihrer Kompetenz, fühlten sich oft hilflos und ohnmächtig. In der Supervisionssitzung wurde ihr Erleben in den Gruppen auf einer Wandzeitung zusammengetragen Überrascht stellten sie fest, wieviel Ähnlichkeit es im Erleben in Gruppen mit diesem Störungsprofil gibt. Ihre Befindlichkeiten konnten mit Hilfe eines kurzen Hinweises zur Psychodynamik der essentiellen Hypertonie auf die der Gegenübertragungsreaktionen zugrundeliegende Störungsdynamik zurückgeführt werden. Sie hatten u.a. das für Hypertoniker typische hohe Anspruchsniveau für sich selbst übernommen. Das bei Hypertonikern selten integrierte aggressive Potential wirkte sich destruktiv auf die Gruppenkohäsion und die Offenheit in den Sitzungen aus. Die Kursleiterinnen fühlten sich nahezu mit einem Maulkorb versehen, der sie hindern sollte, Konfliktpotentiale zu zentrieren, anzusprechen und gar zu bearbeiten.

Neben der Deutung, die als zentrale Methodik der tiefenpsychologisch orientierten Supervision angesehen werden kann, wendet die Supervisorin auch andere nicht deu- 
tende Techniken an. Diese dienen vor allem der Stärkung der Ich-Funktion. Sie sollen dem regressiven Sog, der stets von Gruppen ausgeht, entgegenwirken. Supervisorinnen erklären und begründen zweckmäßigerweise ihre Interventionsschritte, helfen bei der fachlichen Klarifikation, beantworten Rückfragen, greifen aktiv ein, wenn die Arbeitsfähigkeit der Gruppe bezogen auf ihr Arbeitsziel bedroht ist, u.v.m. Es braucht auch einen eher zupackenden, aktivierenden Supervisionsstil, den ich für den supervisorischen Prozess in der Gesundheitsförderung als Haltung der Wahl beschreiben möchte. Supervision darf auf keinen Fall missbräuchlich zu einem therapeutischen Geschehen werden. Ein alternatives, günstiger erscheinendes Verhalten der Kursleiterinnen kann im Rollenspiel erarbeitet werden. Eine Kursleiterin kann z.B. von anderen Supervisonsteilnehmerinnen gedoppelt werden und sich selbst auf diese Weise aus der exzentrischen Position wahrnehmen. Eigene wenig förderliche Verhaltensweisen werden auf diese Weise schnell zugänglich. Imaginationsübungen helfen, die nächste Sitzung vorzubereiten. Ein innerer Rollentausch mit einzelnen Kursteilnehmerinnen oder der Gesamtgruppe lassen die Abwehrstrukturen deutlich werden und den Widerstand schmelzende Interventionen entwickeln. Der methodischen Kreativität der Supervisorin sind keine Grenzen gesteckt. Theoretische Inputs, z.B. zur Analyse von Gruppenprozessen, haben sich bewährt. Die Supervisandinnen können diese Modelle in der Sitzung auf ihre jeweiligen Gruppenkonstellationen anwenden, d.h. sich in konkreter Gruppendiagnostik üben. Möglichst konkrete Handlungsstrategien sollten das Ergebnis von Supervisionsprozessen in der Gesundheitsförderung sein, damit Supervision nicht zum „Psychogesäusel“" verkommt und die Effizienz der Arbeit der „Gesundheitsbildnerinnen“ gewährleistet wird.

Abschließend bleibt mir zu sagen, dass Supervision in der Gesundheitsförderung ein wichtiges Merkmal der Qualitätssicherung ist. Die Möglichkeiten der Prävention, die die Gesundheitsförderung bereitzustellen in der Lage ist, werden m.E. nicht voll ausgeschöpft,

- wenn die Fortbildung der Mitarbeiterinnen durch Supervision nicht gewährleistet ist und die Kursleiterinnen mit der oft schwer geschädigten Klientel überfordert sind und die Gefahr droht, an Stelle qualifizierter Interventionen ,herumzuwurschteln";

- wenn die psychohygienische Funktion von Supervision unterschätzt wird, d.h. wenn eine Identitätsstiftung in der Rolle als Kursleiterin unterbleibt und dadurch eine geringere Arbeitseffizienz, z.B. in Fragen der Motivation und der Tragfähigkeit von Gruppenprozessen, unterbleibt;

- wenn die Rückbindung supervisorischer Arbeitsergebnisse nicht Teil der Organisationsentwicklung wird.

\section{Zusammenfassung}

Nach einer kurzen Spezifizierung des Gegenstandsbereichs „Supervision“ werden die Besonderheiten supervisorischen Tuns im prätherapeutischen Feld der Gesundheitsförderung aufgezeigt. Der Supervision kommt hier sowohl eine identitätsstiftende als auch eine didaktische Funktion für die Kursleiterinnen zu. Anhand von kurzen Sequenzen aus Gruppensupervisionen wird die tiefenpsychologische Methodik veranschaulicht. Im supervisorischen Prozess zeigen sich sowohl Spiegelphänomene der Psychodynamik der Kursteilnehmerinnen als auch die institutionelle Dynamik (ungeklärte Fragen und Konfliktfelder) und deren Verschränkungen. Supervision in der Gesundheitsförderung dient der Qualitätssicherung der Arbeit, der Psychohygiene der Kursleiterinnen und der Organisationsentwicklung. 
Abstract: Supervision in mental-health

A brief outline of supervision as such is followed by a specification of supervision in the pre-therapeutic field of mental-health. Here, supervision has an identity promoting function as well as a didactic one for the leaders of workshops and seminars. Using short sequences of group supervision, the psychoanalytic method is demonstrated. In the course of the supervisory process mirrror phenomena of the participants` psychodynamics can be observed as well as the dynamic of the employing institution (open questions and conflicts) and their interference become visible. Supervision in mental-health is useful for securing the quality of the standards of the work, serves the mental hygiene of the leaders, and promotes the development of the organisation.

Key words: Supervision, mental-health, psychoanalysis, integrative supervision.

\section{Literatur}

Argelander, H. (1970): Das Erstinterview in der Psychotherapie. Darmstadt: Wissenschaftliche Buchgesellschaft.

- (1980): Die Struktur der „Beratung unter Supervision“. Psyche 34, 55-77.

Balint, M. (1955): Psychotherapeutische Ausbildung des praktischen Arztes. Psyche 9, 370-389.

Becker, H. (Hg.)(1995): Psychoanalytische Teamsupervision. Göttingen: Vandenhoeck \& Ruprecht.

Caemmerer, D. v. (Hg.)(1970): Praxisberatung (Supervision). Ein Quellenband. Freiburg

Conrad, G., Pühl, H. (1983): Team-Supervision. Gruppenkonflikte erkennen und lösen. Berlin: Marhold.

Gaertner, A. (1988): Supervision. In: D. Kreft, I. Mielentz (Hg), Wörterbuch sozialer Arbeit (S. 563-565). Weinheim: Beltz.

Haesler, L. (1996): Der angemessene Abstand in der Beziehung zwischen Supervisor und Kandidat. Psyche 4, 321-336.

Kadushin, A. (1990): Supervision in der Sozialarbeit. Supervision 18, 4-24.

Kutter, P. (1992): Das direkte und indirekte Spiegelphänomen. In: H. Pühl (Hg.), Handbuch der Supervision (S. 291-302). Berlin: Edition Marhold.

Lorenzer, A. (1970): Sprachzerstörung und Rekonstruktion. FrankfurtM.: Suhrkamp.

Möller, H. (1998): Schamerleben in Supervisionsgruppen. Gruppendynamik 29 (4), 403-419.

Pollak, T. (1995): Zur Methodik und Technik psychoanalytischer Teamsupervision. In: H. Becker (Hg.), Psychoanalytische Teamsupervision (S. 51-79). Göttingen: Vandenhoeck \& Ruprecht.

Pühl, H. (1992): Der Supervisor als Lehrer und Leiter. In: H. Pühl (Hg.), Handbuch der Supervision I (S. 22-34). Berlin: Edition Marhold.

Schreyögg, A. (1991): Supervision - ein integratives Modell. Paderborn: Junfermann.

- (1995): Coaching. Eine Einführung für Praxis und Ausbildung. FrankfurtM.: Campus.

Die Autorin Heidi Möller, Diplom-Psychologin, PD, Dr. phil., Lehrtherapeutin (FPI, BDP) und Lehrsupervisorin (DGSv, BDP) in freier Praxis, Hochschulassistentin an der TU Berlin, Klinische Psychologie; Forschungsschwerpunkte: Kriminologie, Psychotherapie- und Supervisonsforschung. Anschrift: Technische Universität Berlin, Psychologie im Institut für Sozialwissenschaften, Sekr. HAD 40, Hardenbergstr. 4-5, 10623 Berlin. 